\title{
Phase Correction for Coherent Noise Reduction in Short-Range Radar Measurements
}

\author{
S.G. Beaven ${ }^{1}$, S.P. Gogineni ${ }^{2}$, and P. Kanagaratnam ${ }^{2}$ \\ ${ }^{1}$ Naval Command, Control and Ocean Surveillance Center \\ Research, Development, Test \& Evaluation Division \\ 52111 Mammal Road, San Diego, CA 92152-7670 \\ Phone: (619) 553-4186 * FAX: (619) 553-5366 \\ Email: sbeaven@nosc.mil \\ ${ }^{2}$ Radar Systems and Remote Sensing Laboratory \\ University of Kansas Department of Electrical and Computer Engineering \\ 2291 Irving Hill Road \\ Lawrence, Kansas 66045-2969 \\ Phone: (913) 864-7734 * FAX: (913) 864-7789 \\ Email: gogineni@rsl.ukans.edu
}

\begin{abstract}
Short-range scatterometer systems are used to obtain radar backscatter signatures for understanding the interaction between electromagnetic energy and geophysical media in a number of remote sensing applications. Unlike long-range and intermediate-range radar systems, the sensitivity of short-range radars is not limited by thermal noise, but rather by reflections and leakage signals from the antenna and RF section[1]. These leakage signals and their sidelobes are the primary sources of unwanted signals (coherent noise) in short-range FM radar systems. We have employed coherent noise reduction techniques to reduce the effects of these unwanted signal sources. Reduction of these coherent noise sources is critical to obtaining accurate backscatter measurements from geophysical targets. Recent advances include the use of phase correction to overcome limitations due to drift of local oscillators and effects of temperature changes on the system. Here we present results from the standard and phase-corrected coherent noise reduction techniques. These techniques increased the dynamic range of field and laboratory measurements of radar backscatter from sea ice and allows us to use data from past experiments that had previously been discarded due to low signal-to-noise ratio.
\end{abstract}

\section{INTRODUCTION}

Typically, measurements in which no target is present, or sky measurements, are used to determine coherent noise sources in FM scatterometer data. These measurements often are not feasible when performing measurements in the field because of limitations in the experiment set-up. Furthermore, time taken to perform sky measurements reduces experiment time that is better spent observing backscatter changes that may occur on short time scales due to rapid geophysical changes. In addition, the movement of the radar system to obtain these measurements often results in flexing of the cables used to couple the radar system with the antenna. This reduces the effectiveness of noise reduction with sky measurements.

We have developed coherent noise reduction techniques that use only the measurements from the distributed geophysical targets, and do not require sky measurements. These techniques rely on the property that signals from distributed targets vary spatially and temporally, whereas returned signals from systematic sources do not vary spatially, and only vary slowly with time. We have also added a phase correction method to account for variations in the phase of the systematic noise sources. This extends the noise reduction method to apply to systems whose phase stability is not optimum, and situations in which measurements are obtained over long periods of time. The coherent noise reduction technique, with phase correction reduced systematic noise sources by as much as $50 \mathrm{~dB}$ in experiments performed in the field. These methods have allowed us to process data obtained from past experiments of radar backscatter from sea ice that had been previously discarded due to low signal-to-noise ratio.

\section{COHERENT NOISE REDUCTION (CNR)}

Since 1992 we have used a coherent noise reduction (CNR) technique to process scatterometer data. This technique is described in detail in previous work [2]. Essentially, the algorithm capitalizes on the fact that unwanted systematic reflections within the system are phase coherent, whereas reflections from the target of interest are not coherent from sample to sample. The method uses only 
samples obtained from the measurements from the geophysical target of interest (e.g. sea ice) and do not require special calibration. The only limitation is that the system must be coherent over the period of time in which measurements are used to compute the coherent noise sources. The performance of the CNR technique is limited by the amplitude and phase fluctuations which occur in any system due to temperature effects or movement of cables. Typical phase and amplitude shifts have been shown to be capable of significantly degrading the performance of the standard CNR [3]. The coherent noise reduction algorithm previously used has been successful for most data sets analyzed, but is subject to these limitations.

\section{PHASE-CORRECTED CNR (PC-CNR)}

Significant improvement in the CNR method can be obtained by introducing a phase correction to the process. The phase drift from the systematic noise sources is estimated for each sample to be used. Each measurement is corrected by this phase to force the systematic noise sources to be fully coherent. This requires a dominant systematic noise source, such as the primary antenna reflection, which is ubiquitous in FM scatterometer measurements.

The technique to correct for phase variations between measurements consists of five steps. First, one of the radar return signals is designated the reference signal $\left(v_{0}\right)$ and subsequent return signals $\left(v_{i}\right)$ are phase corrected to this. Second, a prominent coherent reflection (usually the primary antenna reflection) is located in the IF spectrum of $v_{0}$ and designated as the reference spike. Third, both the reference signal and each $v_{1}$ are filtered around the reference spike. Fourth, the phase difference between the filtered signals is determined. Fifth, the return signal $v_{1}$ is advanced in phase according to the phase difference measured to correct for phase errors.

The phase-corrected coherent noise reduction (PC-CNR) process was applied to data obtained with a C-band FM radar systems and a recently developed wideband, plane-wave step-frequency radar [4]. Results of PC-CNR for both radar systems are presented below.

\section{PC-CNR RESULTS}

The phase-corrected coherent noise reduction process (PC-CNR) was applied to data obtained with FM radar systems during a field experiment in 1991 and to data obtained during experiments at the US Army Cold Regions Research and Engineering Laboratory (CRREL) experiments between 1988 and 1995. We've also applied an extension of the technique to data from the wideband plane wave system used during CRREL'95.
The result for the phase-corrected, coherent noise reduction, applied to FM radar data is shown in Fig. 1a as the solid curve, with the original spectrum (no CNR) as the dashed curve. Here each of the coherent noise signals is reduced by approximately $25 \mathrm{~dB}$, and the IF spectrum is clearly improved. The true test of the improvement, though, is illustrated by a direct comparison of the PC-CNR process with the standard CNR process for these data in Fig. Ib. Here we observe that the standard CNR suppresses the noise spikes by less than $5 \mathrm{~dB}$. This is caused by the fact that the data were obtained over a long period of time (nearly 24 hours), and any of a number of sources of phase drift have resulted in a degradation of the CNR process. However, the PC-CNR result has reduced the coherent portions of the spectrum by an additional 10 to $15 \mathrm{~dB}$. The improvement of PC-CNR over standard CNR may be better illustrated on a linear scale as depicted in Fig. 2. Here it is clear that the return from the target (sea ice) at a range of approximately 23 $\mathrm{m}$ is the dominant return after phase-correction. The standard CNR does not significantly reduce the unwanted noise spikes for these data.

We also applied the PC-CNR process for wideband data to measurements obtained during CRREL'95 and the results are shown in Fig. 3. These results demonstrate that the PCCNR improves the measurement over the standard CNR. The PC-CNR improves the SNR in the vicinity of the surface return by 20 to $25 \mathrm{~dB}$.

\section{CONCLUSIONS}

We have developed coherent noise reduction (CNR) and phase-corrected coherent noise reduction (PC-CNR) techniques to improve radar backscatter measurements with short-range FM and step-frequency radar systems. Shortrange radar systems are not limited by thermal noise, but by the leakage and reflection signals from the antenna and RF sections. The coherent noise reduction techniques developed here reduce the effects of these leakage and reflection signals. These schemes hinge on the fact that the returns from distributed targets are non-coherent from sample to sample and that the returns from systematic sources are coherent from sample to sample. Phase-correction algorithms for improving the CNR process in the presence of phase variations caused by oscillator drift, cable flexing or temperature fluctuations were also developed. These algorithms result in measurements whose sensitivity approaches the thermal noise floor, rather than being limited by systematic leakage and reflection signals.

\section{ACKNOWLEDGMENTS}

This work was sponsored in part by Office of Naval Research (ONR) grant \#N00014-89-J-1456 and an ONR Doctoral Fellowship. 


\section{REFERENCES}

[1] W.K. Saunders, CW and FM Radar, in Radar Handbook, edited by M. Skolnik (New York:McGraw-Hill), 14.1$14.45,1990$.

[2] S. Beaven, and S.P. Gogineni, "Shipborne Radar Backscatter Measurements from Arctic Sea Ice During the Fall Freeze-Up," Remote Sensing Reviews, vol. 9, nos. 1-2, pp. 3-25, 1994.

[3] S.G. Beaven, "Sea Ice Radar Backscatter Modeling, Measurements, and the Fusion of Active and Passive Microwave Data," Ph.D. Dissertation, University of Kansas, 1995.

[4] Gogineni, S.P., K. Jezek, L. Peters, J. Young, S. Beaven, and E. Nassar, Application of plane waves for accurate measurement of microwave scattering from geophysical surfaces, IEEE Transactions on Geoscience and Remote Sensing, May, 1995.

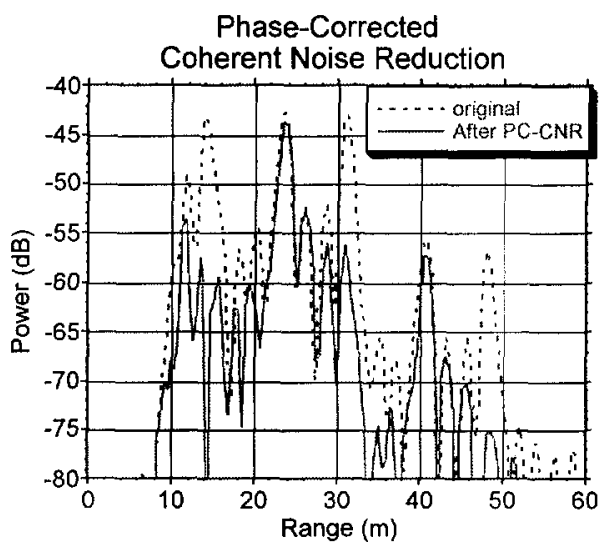

a)

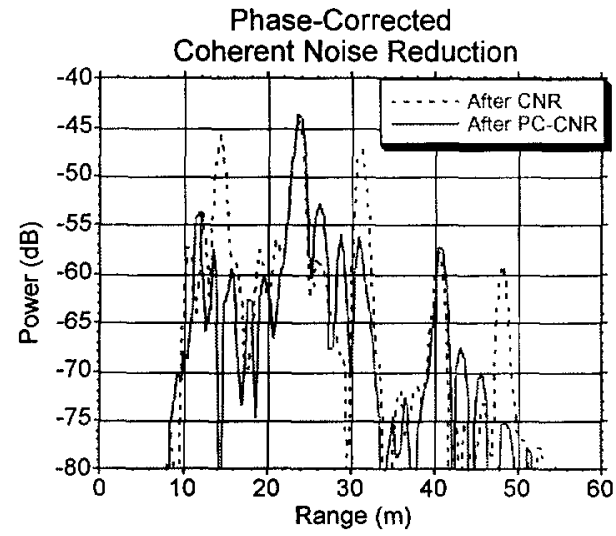

b)

Fig. 1. Result of coherent noise reduction with phase correction. (a) The corrected IF spectrum is plotted along with the original to demonstrate the suppression of unwanted coherent noise spikes. (b) Result of CNR with phase correction, compared to CNR without phase correction.

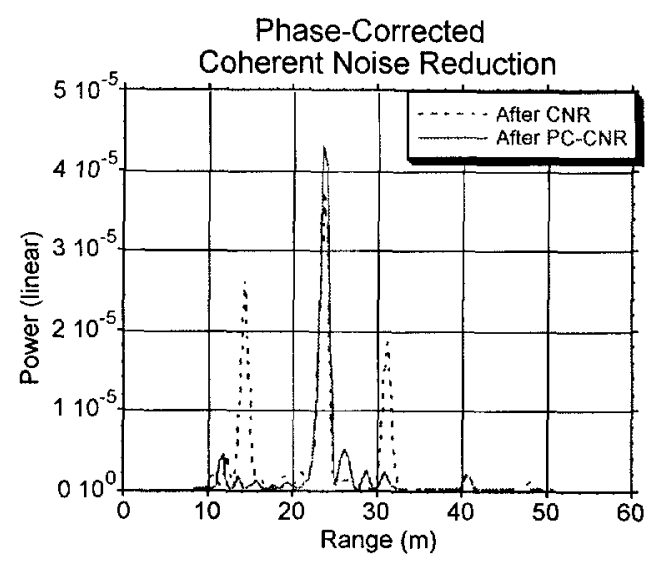

Fig. 2. Result from Fig. 1a, plotted on a linear scale to illustrate the suppression of coherent noise in the IF spectrum

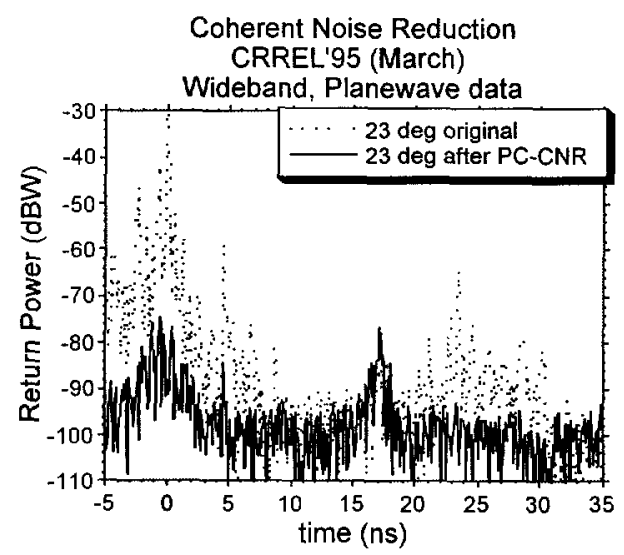

Fig. 3. PC-CNR for wideband, step-frequency radar measurements, compared to the original spectrum The antenna reflection is reduced by $50 \mathrm{~dB}$, whereas standard CNR (not shown) resulted in 25-dB suppression. 